TRANSACTIONS OF THE

AMERICAN MATHEMATICAL SOCIETY

Volume 352, Number 10, Pages 4711-4730

S 0002-9947(00)02608-8

Article electronically published on June 8, 2000

\title{
PERIODIC POINTS OF HOLOMORPHIC MAPS VIA LEFSCHETZ NUMBERS
}

\author{
NÚRIA FAGELLA AND JAUME LLIBRE
}

\begin{abstract}
In this paper we study the set of periods of holomorphic maps on compact manifolds, using the periodic Lefschetz numbers introduced by Dold and Llibre, which can be computed from the homology class of the map. We show that these numbers contain information about the existence of periodic points of a given period; and, if we assume the map to be transversal, then they give us the exact number of such periodic orbits. We apply this result to the complex projective space of dimension $n$ and to some special type of Hopf surfaces, partially characterizing their set of periods. In the first case we also show that any holomorphic map of $\mathbb{C} P(n)$ of degree greater than one has infinitely many distinct periodic orbits, hence generalizing a theorem of Fornaess and Sibony. We then characterize the set of periods of a holomorphic map on the Riemann sphere, hence giving an alternative proof of Baker's theorem.
\end{abstract}

\section{INTRODUCTION}

In dynamical systems and, in particular, in the study of iteration of self maps of a given manifold, periodic orbits play an important role.

Given a continuous map $f: X \rightarrow X$, a point $x \in X$ is called periodic if there exists $k \in \mathbb{N}$ such that $f^{k}(x)=x$. The minimum of such $k$ is called the period of $x$, and the iterates $\left\{x, f(x), \ldots, f^{k-1}(x)\right\}$ form a periodic orbit. For such a map, it is natural to ask how many periodic orbits it has or what are the possible periods that may appear. To deal with these problems, differential topological methods have often proved to be very useful, since it is clear that the topology of the manifold in question plays an essential role.

The Lefschetz Fixed Point Theorem was one of the main results in this direction. Knowing the homology class of the map, one can compute its Lefschetz number $L(f)$ and, if the result is nonzero, conclude the existence of a fixed point. Clearly, the same process, applied to the $k^{\text {th }}$ iterate of the function, $f^{k}$, would give the existence of a periodic orbit of period $k$, or a divisor of $k$. We have gone a long way from this theorem, and there is plenty of literature on its generalizations and applications (see [2, 5, 8, 16]).

To deal with the problem of existence of periodic orbits with a given period the periodic Lefschetz number of period $k$, denoted by $l\left(f^{k}\right)$, was introduced in

Received by the editors October 28, 1998.

2000 Mathematics Subject Classification. Primary 55M20; Secondary 32H50, 37B99.

Key words and phrases. Set of periods, periodic points, holomorphic maps, Lefschetz fixed point theory.

Both authors are partially supported by DGICYT grant number PB96-1153. 
[7] and [15]. These numbers are defined for any continuous map but, in general, they become really interesting when we restrict to the set of transversal functions. Recall that a periodic point $x$ of period $k$ of a $\mathcal{C}^{1}$ map $f$ is nondegenerate if $\operatorname{det}\left(\operatorname{Id}-d f^{m k}(x)\right) \neq 0$ for all $m \in \mathbb{N}$. We say that $f$ is transversal if all its periodic points are nondegenerate. For a transversal $\mathcal{C}^{1}$ map on a compact manifold, $l\left(f^{m}\right) \neq 0$ implies the existence of a periodic orbit of period $m$ if $m$ is odd, and period $m$ or $m / 2$ if $m$ is even (see [15] and [12]).

In this paper we deal with maps $f: M \rightarrow M$ where $M$ is a compact complex manifold and $f$ is holomorphic. We will always assume that the periodic points of $f$ are isolated in the set $\operatorname{Fix}\left(f^{m}\right)$ for all $m$, where

$$
\operatorname{Fix}\left(f^{m}\right)=\left\{x \in M \mid f^{m}(x)=x\right\} .
$$

For a holomorphic map this is not a strong assumption. Indeed, if that were not the case, $\operatorname{Fix}\left(f^{m}\right)$ would be a variety of (complex) dimension at least one (see for example 22]). Hence we consider this a degenerate case.

D. Fried in [9] worked in this setting and gave criteria for these maps to have infinitely many periodic orbits. Our goal is to study the periodic structure of these maps. We will see that the rigidity of holomorphic maps makes the periodic Lefschetz numbers a much more powerful tool than in the $\mathcal{C}^{1}$ case, since one can remove the hypothesis on the oddity of the period $m$.

Let

$$
\operatorname{Per}(f)=\{m \in \mathbb{N} \mid f \text { has a periodic orbit of period } m\},
$$

where, as usual, $\mathbb{N}$ denotes the set of positive integers.

In Section 3 we will prove (see Theorem 3.1)

Theorem A. Let $M$ be a compact complex manifold and $f: M \rightarrow M$ a nonconstant holomorphic map. Then, there exists $M>0$ such that, for all $p \in \mathbb{N}$ prime and $p>M, l\left(f^{p}\right) \neq 0$ if and only if $p \in \operatorname{Per}(f)$. If furthermore $f$ is transversal, then, for all $m \in \mathbb{N}, l\left(f^{m}\right)$ is the number of periodic points of period $m$.

We observe also that in some cases the use of these numbers can be twofold: computing the periodic orbits of a few low periods we obtain some Lefschetz numbers from which we can obtain the homology class of the functions in question, something that in general it is not easy to compute.

In Section 4 we apply Theorem A to two different examples. The first application deals with the complex projective space $\mathbb{C} P(n)$. For holomorphic maps of $\mathbb{C} P(2)$ Fornaess and Sibony in [10] used complex analytical techniques to show that they have infinitely many periodic orbits. Using a criterium of [19] and Theorem A, we will show the following. (see Theorem 4.2):

Theorem B. Any holomorphic map $f: \mathbb{C} P(n) \rightarrow \mathbb{C} P(n)$ of degree $d \geq 2$ has infinitely many periodic orbits. If furthermore we assume $f$ to be transversal, then $f$ has periodic orbits of all periods.

The second application (Section 4.2) deals with some special type of Hopf surfaces, for which we first characterize their holomorphic self maps and then show a similar theorem.

For general holomorphic maps, that is, not necessarily transversal, the complete characterization of the set of periods becomes very complicated. To our knowledge, the set of possible periods (of holomorphic maps on compact complex manifolds) 
has only been studied for the 2-dimensional sphere by Baker in [3. Using complex analytical techniques, he showed (see Theorem [5.1) that any holomorphic map of $\mathbb{C} \cup \infty$ must have periodic orbits of all periods except, maybe, periods 2,3 or 4 . In Section 5 we give a different proof of Baker's theorem using the periodic Lefschetz numbers. The theorem becomes a corollary of the following (see Theorem 5.1).

Theorem C. Let $f$ be a rational map of $\mathbb{C}$ of degree $d$. If $l\left(f^{m}\right)>2 m(d-1)$, then $m \in \operatorname{Per}(f)$.

This result makes heavy use of the strong relation between degenerate periodic points and critical points of $f$ (points of derivative 0 ). This is a special property of one dimensional holomorphic dynamical systems; hence we think it will be difficult to generalize this technique to higher dimensional systems. Using a somewhat different approach, work in this direction has been done by Fried in [9].

The authors would like to thank P. Ahern, D. Fried, A. Gasull and F. Mañosas for helpful conversations.

\section{LEFSCHETZ NUMBERS}

The key work of Lefschetz in the 1920's was to relate the homology class of a given map with the earlier work of Brouwer on indices of self-mappings of manifolds. These two contexts give two equivalent definitions for the Lefschetz numbers, and from their comparison, one obtains the information about fixed points.

Let $f: M \rightarrow M$ be a continuous map and $M$ a compact manifold of dimension $n$. Then, $f$ induces homomorphisms on the simplicial homology groups with rational coefficients

$$
f_{* i}: H_{i}(M, \mathbb{Q}) \longrightarrow H_{i}(M, \mathbb{Q}), \quad 0 \leq i \leq n,
$$

where, as usual, $\mathbb{Q}$ denotes the set of rational numbers. These groups are finite dimensional, and hence, once we choose a basis, each of the homomorphisms $f_{* i}$ may be written as a matrix with respect to this basis. The first definition of the Lefschetz number of $f: M \rightarrow M$ as above is

$$
L(f)=\sum_{i=0}^{n}(-1)^{i} \operatorname{trace}\left(f_{* i}\right) .
$$

On the other hand, let $x \in M$ be an isolated fixed point of $f$. If $B$ is a small closed ball centered at $x$ that contains no other fixed point of $f$, then the assignment

$$
z \mapsto \frac{f(z)-z}{|f(z)-z|}
$$

defines a smooth map $F: \partial B \rightarrow S^{n-1}$, where $S^{n-1}$ is the sphere of dimension $n-1$. The degree of this map is called the (Poincaré) index of $f$ at $x$, and we denote it by ind $(f, x)$. On the plane, the Poincaré index is the winding number of the vector field $f(z)-z$ about the fixed point $x$. Equivalently, one can think of the Poincaré index as the local degree of $f$-Id at the fixed point $x$, i.e. the number of solutions of $f(z)-z=\varepsilon$ in $B$ (each one with a plus or minus sign depending on whether $f$-Id preserves or reverses orientation at that point), where $\varepsilon \in M$ is sufficiently close to zero and a regular value of $f$-Id. It is important to note that the Poincaré index is constant under small perturbations of the map (see [5] 6]). 
In this context, the Lefschetz number can also be defined as follows. Let $f$ : $M \rightarrow M$ be as above, and assume all its fixed points are isolated. Then,

$$
L(f)=\sum_{x \in \operatorname{Fix}(f)} \operatorname{ind}(f, x) .
$$

The assumption on $f$ having only isolated fixed points is not important, since $L(f)$ only depends on the homotopy class of $f$ and any map of $M$ is arbitrarily closed to a homotopic map with isolated fixed points.

Now, this implies that $L(f)$ is always an integer and leads to the celebrated Lefschetz Fixed Point Theorem: If $L(f) \neq 0$, then $f$ has a fixed point. In consequence, if $L\left(f^{m}\right) \neq 0$ then $f$ has a periodic orbit of period a divisor of $m$, i.e. not necessarily of period $m$. The converse of the theorem is not true.

The growth of the sequence $\left\{L\left(f^{m}\right)\right\}_{m \in \mathbb{N}}$ contains plenty of information about the growth of the periodic points. This is a consequence of the following fact.

Theorem 2.1 (Shub and Sullivan, [19]). Suppose that $f: \mathbb{R}^{n} \rightarrow \mathbb{R}^{n}$ is $\mathcal{C}^{1}$ and that $x$ is an isolated fixed point of $f^{m}$ for all $m$. Then the sequence $\left\{\operatorname{ind}\left(f^{m}, x\right)\right\}_{m \in \mathbb{N}}$ is bounded.

Remark 2.2. In fact, looking closely at Shub and Sullivan's proof, one sees that they give a complete description of this sequence as follows. Suppose $m$ is a multiple of $k_{1}, \ldots, k_{p}$, where these are orders of some of the roots of unity that appear as eigenvalues of $d f(x)$. Suppose also that $m$ is not a multiple of $k_{p+1}, \ldots, k_{s}$, where these are the orders of the remaining roots of unity that appear as eigenvalues of $d f$. Then, they show that $\operatorname{ind}\left(f^{m}, x\right)= \pm \operatorname{ind}\left(f^{\lambda}, x\right)$, where $\lambda=\operatorname{lcm}\left(k_{1}, \ldots, k_{p}\right)$. Hence, the sequence $\left\{\left|\operatorname{ind}\left(f^{m}, x\right)\right|\right\}_{m \in \mathbb{N}}$ is periodic.

We will use the following corollary in Section 4. If $A$ is a finite set, let \# $A$ denote its cardinality.

Corollary $2.3(\sqrt{19})$. Let $f$ and $x$ be as in Theorem [2.1. If the sequence of Lefschetz numbers $\left\{L\left(f^{m}\right)\right\}_{m \in \mathbb{N}}$ is unbounded, then $f$ has infinitely many periodic orbits.

Proof. Let PP denote the set of periodic points of $f$, which we assume to be finite. For each $x \in \mathrm{PP}$ we let $p(x)$ be its period. Then, we can define

$$
C=\max _{x \in \mathrm{PP}} \max _{k \in \mathbb{N}} \operatorname{ind}\left(f^{k p(x)}, x\right) .
$$

It follows then that

$$
L\left(f^{m}\right)=\sum_{x \in \operatorname{Fix}\left(f^{m}\right)} \operatorname{ind}\left(f^{m}, x\right) \leq C(\# \mathrm{PP})
$$

for all $m \in \mathbb{N}$.

Although the Lefschetz numbers contain information about the growth of periodic orbits, they cannot be used to study the existence of periodic points of a given period. To deal with this problem the periodic Lefschetz numbers were introduced in 7 and 15. The Lefschetz number of period $m$ is defined as

$$
l\left(f^{m}\right)=\sum_{r \mid m} \mu(r) L\left(f^{\frac{m}{r}}\right),
$$


where $\mu$ is the Möbius function given by

$$
\mu(r)= \begin{cases}1 & \text { if } r=1 \\ 0 & \text { if } k^{2} \mid r \text { for some } k \in \mathbb{N} \\ (-1)^{k} & \text { if } r=p_{1} \cdots p_{k} \text { (distinct prime factors) }\end{cases}
$$

By the Jacobi inversion formula (see for example [17]),

$$
L\left(f^{m}\right)=\sum_{r \mid m} l\left(f^{r}\right)
$$

The periodic Lefschetz numbers become really interesting when we restrict to the class of transversal maps, i.e., maps whose periodic points are nondegenerate. If the graph of a transversal map intersects the diagonal of $M \times M$ it does so transversely; hence all periodic points of a given period are isolated.

For fixed points of transversal maps, the Poincaré index can only be 1 or -1 , depending on whether $f$ preserves or reverses orientation at that point. That is, if $x$ is a fixed point of $f^{m}$,

$$
\operatorname{ind}\left(f^{m}, x\right)=\operatorname{sgn}\left(\operatorname{det}\left(I-\mathrm{d} f^{m}(x)\right)\right)=(-1)^{u_{+}(x)},
$$

where $u_{+}(x)$ is the number of real eigenvalues of $d f^{m}(x)$ that are strictly larger than one (see for instance [8]). This fact is essential in the proof of the following theorem.

Theorem 2.4 (see [15] and [12]). Let $f: M \rightarrow M$ be a transversal map on a compact manifold. Given $m \in \mathbb{N}$, if $l\left(f^{m}\right) \neq 0$ then

(a) $m \in \operatorname{Per}(f)$ if $m$ is odd,

(b) $\{m, m / 2\} \cap \operatorname{Per}(f) \neq \emptyset$ if $m$ is even.

We include the idea of the proof for completeness as well as for its later use in Section 5

Sketch of the proof. The Lefschetz zeta function of $f$ is defined formally as

$$
Z_{f}(t)=\exp \left(\sum_{m=1}^{\infty} \frac{L\left(f^{m}\right)}{m} t^{m}\right) .
$$

Using the two equivalent definitions of $L\left(f^{m}\right)$, and after some calculations, one has two formal expressions for $Z_{f}(t)$, namely

$$
Z_{f}(t)=\prod_{m=1}^{\infty}\left(1-t^{m}\right)^{-\frac{l\left(f^{m}\right)}{m}}
$$

and

$$
Z_{f}(t)=\prod_{\gamma \in \mathrm{PO}}\left(1-(-1)^{u_{-}(\gamma)} t^{p(\gamma)}\right)^{(-1)^{u_{+}(\gamma)+u_{-}(\gamma)+1}},
$$

where PO is the set of periodic orbits of $f, p(\gamma)$ is the period of $\gamma \in \mathrm{PO}$, and $u_{+}(\gamma)$ (respectively $u_{-}(\gamma)$ ) is the number of real eigenvalues of $d f^{p(\gamma)}(x)$ strictly larger than 1 (respectively strictly smaller than -1 ) for any $x \in \gamma$. Comparing these two expressions, we get the theorem.

We remark that (2.2) holds for any continuous map, while (2.3) holds only for transversal maps. 


\section{The COMPlex SETting}

Let $M$ be a compact complex manifold of complex dimension $n$ and $f: M \rightarrow M$ a holomorphic map. Since $M$ can be understood as a differentiable manifold of real dimension $2 n$, all the definitions and results above on transversal maps can be applied to this case. However, in the complex setting, the Lefschetz numbers give more information about periodic points than in the real setting.

Set

$$
\operatorname{Fix}\left(f^{m}\right)=\left\{x \in M \mid f^{m}(x)=x\right\}
$$

and

$$
\operatorname{Per}_{m}(f)=\{x \in M \mid x \text { is periodic of period } m\} \text {. }
$$

Theorem 3.1. Let $M$ be a compact complex manifold and $f: M \rightarrow M$ a nonconstant holomorphic map. Assume that all periodic points of $f$ are isolated. Then

(a) $L\left(f^{m}\right) \geq \# \operatorname{Fix}\left(f^{m}\right)$, for all $m \in \mathbb{N}$.

(b) $l\left(f^{p}\right) \geq \# \operatorname{Per}_{p}(f)$, for all $p \in \mathbb{N}$ prime.

(c) There exists $M>0$ such that, for all $p \in \mathbb{N}$ prime and $p>M, l\left(f^{p}\right) \neq 0 \Longleftrightarrow$ $p \in \operatorname{Per}(f)$.

(d) If all fixed points of $f$ are nondegenerate, then (c) holds for all $p \in \mathbb{N}$ prime.

(e) If $f$ is transversal, then $l\left(f^{m}\right)=\# \operatorname{Per}_{m}(f)$, for all $m \in \mathbb{N}$.

We remark that (d) only assumes the fixed points to be nondegenerate. As opposed to transversality, this condition can be checked in many cases.

For the proof we need the well-known facts stated in the following lemma.

Lemma 3.2. Let $A$ be an $n \times n$ complex matrix and let $B: \mathbb{R}^{2 n} \rightarrow \mathbb{R}^{2 n}$ be the linear map $A: \mathbb{C}^{n} \rightarrow \mathbb{C}^{n}$ considered in $\mathbb{R}^{2 n}$. Then, any real eigenvalue of $B$ has even multiplicity and $\operatorname{det}(B) \geq 0$.

Proof. With a convenient choice of basis, the matrix $B$ may be written as

$$
B=\left(\begin{array}{cc}
\operatorname{Re} A & -\operatorname{Im} A \\
\operatorname{Im} A & \operatorname{Re} A
\end{array}\right)
$$

If $\lambda$ is a real eigenvalue of $B$ and $v=(x, y) \in \mathbb{R}^{n} \times \mathbb{R}^{n}$ is an eigenvector of eigenvalue $\lambda$, then $(-y, x)$ is a different eigenvector of eigenvalue $\lambda$. Hence the subspace associated with $\lambda$ has even dimension.

To see that $\operatorname{det}(B) \geq 0$ we can change basis to write $B$ in the form

$$
B^{\prime}=\left(\begin{array}{cc}
\operatorname{Re} A+i \operatorname{Im} A & 0 \\
0 & \operatorname{Re} A-i \operatorname{Im} A
\end{array}\right)=\left(\begin{array}{cc}
A & 0 \\
0 & \frac{\bar{A}}{A}
\end{array}\right) .
$$

Hence $\operatorname{det}(B)=\operatorname{det}\left(B^{\prime}\right)=\operatorname{det}(A) \operatorname{det}(\bar{A})=|\operatorname{det} A|^{2} \geq 0$.

Proof of Theorem 3.1. To show (a), notice that a holomorphic map, thought of as a real map, always preserves the orientation at any point. Indeed, if $A=d f^{m}(z)$ for any $z \in M$ and $B$ is defined by $A$ as in Lemma [3.2, it follows that $\operatorname{det}(B) \geq 0$ and hence $f^{m}$ preserves the orientation at all points of $M$. Hence, the local degree of $f$-Id near any point $x \in \operatorname{Fix}\left(f^{m}\right)$ is the multiplicity of this fixed point, which must be positive since the map is nonconstant. We then have

$$
\operatorname{ind}\left(f^{m}, x\right)>0, \text { for all } x \in \operatorname{Fix}\left(f^{m}\right),
$$


and hence

$$
L\left(f^{m}\right)=\sum_{x \in \operatorname{Fix}\left(f^{m}\right)} \operatorname{ind}\left(f^{m}, x\right) \geq \# \operatorname{Fix}\left(f^{m}\right) .
$$

To show the remainder of the theorem we combine de definitions of $L\left(f^{p}\right)$ and $l\left(f^{p}\right)$ for $p$ prime to obtain

$$
\begin{aligned}
l\left(f^{p}\right) & =L\left(f^{p}\right)-L(f) \\
& =\sum_{x \in \operatorname{Fix}\left(f^{p}\right)} \operatorname{ind}\left(f^{p}, x\right)-\sum_{x \in \operatorname{Fix}(f)} \operatorname{ind}(f, x) \\
& =\sum_{x \in \operatorname{Per}_{p}(f)} \operatorname{ind}\left(f^{p}, x\right)+\sum_{x \in \operatorname{Fix}(f)}\left(\operatorname{ind}\left(f^{p}, x\right)-\operatorname{ind}(f, x)\right) .
\end{aligned}
$$

If $x \in M$ is a fixed point of $f$, then $\operatorname{ind}\left(f^{p}, x\right) \geq \operatorname{ind}(f, x)$. Indeed, this is clear since the multiplicity of a fixed point $x$ can only increase with the iterates of the map. Alternatively, one can also think that any zero of $f-\mathrm{Id}-\varepsilon$ has to be also a solution of $(f-\varepsilon)^{p}-\mathrm{Id}$, which is a perturbation of $f^{p}-\mathrm{Id}$. Then from (3.1) we obtain

$$
l\left(f^{p}\right) \geq \sum_{x \in \operatorname{Per}_{p}(f)} \operatorname{ind}\left(f^{p}, x\right) \geq \# \operatorname{Per}_{p}(f)
$$

and (b) follows.

Moreover, from Remark 2.2 it follows that $\operatorname{ind}\left(f^{p}, x\right) \neq \operatorname{ind}(f, x)$ only when $d f(x)$ has an eigenvalue which is a primitive $p^{\text {th }}$ root of unity. Since $f$ has a finite number of fixed points, the number of such roots is finite. Hence, for $p$ large enough, these two indices are equal, and (c) follows.

We now assume that all fixed points are nondegenerate. Let $x \in \operatorname{Fix}(f)$ and $A=d f^{m}(x)$ for any $m \in \mathbb{N}$. Since $f^{m}$ is holomorphic, any real eigenvalue of the real matrix $B$ defined by $A$ as in the statement of Lemma 3.2 has even multiplicity. Since there cannot be any eigenvalue equal to 1 , we may compute the index from (2.1), and we get ind $\left(f^{m}, x\right)=1$ for all $m \in \mathbb{N}$. Therefore, from (3.1) we obtain

$$
l\left(f^{p}\right)=\sum_{x \in \operatorname{Per}_{p}(f)} \operatorname{ind}\left(f^{p}, x\right)
$$

for any prime $p$, hence proving (d).

Finally, to see (e), assume that $f$ is transversal, i.e., that all periodic points are nondegenerate. Then, the argument above holds for any periodic point and not only for the fixed points. Hence, from (2.1) we have ind $\left(f^{m}, x\right)=1$ for all $x \in \operatorname{Fix}\left(f^{m}\right)$ and all $m \in \mathbb{N}$. Therefore the Lefschetz number $L\left(f^{m}\right)$ defined above is exactly the cardinal of the set $\operatorname{Fix}\left(f^{m}\right)$, i.e. the number of periodic points of period some divisor of $m$. Hence

$$
L\left(f^{m}\right)=\sum_{r \mid m} \# \operatorname{Per}_{r}(f) .
$$

By the Jacobi Inversion Formula (see [17]), we have \# $\operatorname{Per}_{r}(f)=l\left(f^{r}\right)$ as defined above.

An immediate corollary of this theorem is the following criteria. 
Corollary 3.3. Let $f$ be as in Theorem [3.1. Then the following statements are equivalent.

(a) The map $f$ has infinitely many periodic points of prime period.

(b) The set of $p \in \mathbb{N}$ prime such that $l\left(f^{p}\right) \neq 0$ is unbounded.

(c) The zeta function $Z_{f}(t)$ has infinitely many factors of the form $1-t^{p}$ for $p$ prime.

We remark that the third part of the corollary comes from expression (2.2) for a zeta function.

V. Arnold in [1 posed the following question: "If $f: M \rightarrow M$ is a (real) analytic diffeomorphism of a compact analytic manifold, is the number of periodic points of period $m$ bounded above by an exponential function of $m$ ?" We remark that, in the special case of a holomorphic map, the answer can be viewed as a corollary of Theorem 3.1 .

Corollary 3.4. Let $M$ be a compact complex manifold and $f: M \rightarrow M$ a holomorphic map. Assume that all periodic points of $f$ are isolated. Then,

$$
\# \operatorname{Fix}\left(f^{m}\right) \leq C \lambda^{m}
$$

for some positive real numbers $C$ and $\lambda$.

Proof. By Theorem 3.1 (a), the number of fixed points of $f^{m}$ is bounded by the Lefschetz number $L\left(f^{m}\right)$. By definition,

$$
L\left(f^{m}\right)=\sum_{i=0}^{2 n}(-1)^{i} \operatorname{trace}\left(f_{* i}^{m}\right),
$$

where $n$ is the complex dimension of $M$. Since for all values of $i$, the matrices $f_{* i}$ are finite dimensional and their traces are the sum of their eigenvalues, we have that

$$
L\left(f^{m}\right)=\lambda_{1}^{m}+\ldots+\lambda_{k}^{m}-\left(\lambda_{k+1}^{m}+\ldots+\lambda_{s}^{m}\right),
$$

where the $\lambda_{j}$ 's are eigenvalues of the matrices $f_{* i}$ and we have done all possible cancellations. Finally, let

$$
\lambda=\max _{1 \leq i \leq s}\left|\lambda_{i}\right|,
$$

and the conclusion follows taking the constant $C=s$.

3.1. Computation of $l\left(f^{m}\right)$. In this section we include some criteria that allow us to determine when the periodic Lefschetz numbers are different from zero. In general it is not easy to find an explicit expression for $l\left(f^{m}\right)$ in terms of $m$, though, in some examples, it is easy to do so for $L\left(f^{m}\right)$. Clearly, the growth of the Lefschetz numbers contains a lot of information about the periodic Lefschetz numbers. For the remainder of this section we assume $f$ to be a continuous map from a compact manifold $M$ to itself, where $M$ has dimension $n$. We will use the following proposition in the two applications of Section 4 .

Proposition 3.5. Suppose that $f$ is a continuous map from a compact manifold $M$ to itself and it satisfies

$$
d^{k m}<L\left(f^{m}\right)<d^{k m+1}
$$

for some $d \geq 2, k \geq 1$ and for all $m \in \mathbb{N}$. Then, $l\left(f^{m}\right)>0$ for all $m \geq 3$. 
For the proof we need the following lemma.

Lemma 3.6. The polynomials

$$
P_{n}(d)=d^{n}-d^{n-1}-\ldots-1
$$

satisfy $P_{n}(d)>0$ for all $d \geq 2$ and for all $n \geq 1$.

Proof. Clearly

$$
1+d+d^{2}+\ldots+d^{n-1}=\frac{d^{n}-1}{d-1}<d^{n}
$$

and we are done.

Proof of Proposition 3.5. Let $P_{n}(d)$ be as in Lemma 3.6.

Let $m=p_{1}^{\alpha_{1}} \cdots p_{s}^{\alpha_{s}}$, where $p_{1}, \ldots, p_{s}$ are distinct prime numbers. Then, by definition of $l\left(f^{m}\right)$,

$$
\begin{aligned}
& l\left(f^{m}\right)=\sum_{r \mid m} \mu(r) L\left(f^{\frac{m}{r}}\right) \\
& =L\left(f^{m}\right)-\sum_{1 \leq i \leq s} L\left(f^{\frac{m}{p_{i}}}\right)+\sum_{1 \leq i<j \leq s} L\left(f^{\frac{m}{p_{i} p_{j}}}\right)-\cdots+(-1)^{s} L\left(f^{\frac{m}{p_{1} \cdots p_{s}}}\right) \\
& >d^{k m}-\sum_{1 \leq i \leq s} d^{\frac{k m}{p_{i}}+1}+\sum_{1 \leq i<j \leq s} d^{\frac{k m}{p_{i} p_{j}}}-\cdots+(-1)^{s} d^{\frac{k m}{p_{1} \cdots p_{s}}+\frac{1}{2}\left(1+(-1)^{s-1}\right)} \\
& >P_{k m}(d),
\end{aligned}
$$

where the last inequality holds because $k m>\frac{k m}{2}+1$ for all $m \geq 3$ and all $k \geq 1$, together with the fact that $p=2$ is the smallest prime number apart from 1 .

Since $P_{k m}(d)>0$ for all $d \geq 2$, the result follows.

Remark 3.7. The same proof would work if the condition of Proposition 3.5 were $d^{k m-1}<L\left(f^{m}\right)<d^{k m}$.

For completeness we also include a criterium that was proved in [11].

Let $f_{*}$ be the block matrix which has the matrices $f_{* k}$ on the diagonal for $0 \leq$ $k \leq n$, and zeroes elsewhere. Let $\left(f_{*}\right)^{e}$ (respectively $\left.\left(f_{*}\right)^{o}\right)$ be the block matrix which has the matrices $f_{* k}$ for $k$ even (respectively $k$ odd) on the diagonal and zeroes elsewhere. Given a matrix $A$, define its spectral radius $\sigma(A)$ to be equal to the maximum of the absolute values of its eigenvalues.

Theorem 3.8 (11]). Suppose that the limit $\lim _{m \rightarrow \infty}\left|\operatorname{trace}\left(F_{*}^{m}\right)\right|^{1 / m}$ exists for $F_{*} \in\left\{\left(f_{*}\right)^{e},\left(f_{*}\right)^{o}\right\}$, and that $\sigma\left(\left(f_{*}\right)^{e}\right) \neq \sigma\left(\left(f_{*}\right)^{o}\right)$. If there is an eigenvalue of $f_{*}$ different from a root of unity and zero, then there exists $M$ such that $l\left(f^{p}\right) \neq 0$ for all $p \in \mathbb{N}$ prime, $p>M$.

Furthermore, if we assume that the limit $\lim _{m \rightarrow \infty}\left|\sum_{r \mid m} \mu(r) \operatorname{trace}\left(F_{*}^{m / r}\right)\right|^{1 / m}$ exists for $F_{*} \in\left\{\left(f_{*}\right)^{e},\left(f_{*}\right)^{o}\right\}$, then there exists $M>0$ such that $l\left(f^{m}\right) \neq 0$ for all $m>M$.

\section{Applications}

4.1. Holomorphic mappings of $\mathbb{C} P(n)$. The complex projective space of dimension $n$ is the quotient of $\mathbb{C}^{n+1}$ by the equivalence relation

$$
\left(z_{0}, z_{1}, \cdots, z_{n}\right) \sim \lambda\left(z_{0}, z_{1}, \cdots, z_{n}\right) \text { for all } \lambda \in \mathbb{C} \backslash\{0\} .
$$


Points in $\mathbb{C} P(n)$ are represented in homogeneous coordinates by

$$
\left[z_{0}: z_{1}: \cdots: z_{n}\right], \quad z_{i} \neq 0 \text { for some } 0 \leq i \leq n
$$

which denotes an equivalence class.

The space $\mathbb{C} P(n)$ is then the natural compactification of $\mathbb{C}^{n}$ and can be thought as adding at infinity a subspace of dimension $n-1$. For example, $\mathbb{C} P(1)$, the Riemann sphere, is the compactification of $\mathbb{C}$ obtained by adding the point at infinity. This makes holomorphic maps of $\mathbb{C} P(n)$ the natural generalization of rational maps of the Riemann sphere.

Holomorphic maps of $\mathbb{C} P(n)$ are characterized as follows.

Theorem 4.1 ([10]). Let $f$ be a nonconstant holomorphic map from $\mathbb{C} P(n)$ to $\mathbb{C} P(n)$. Then, $f$ is given in homogeneous coordinates by $\left[f_{0}: f_{1}: \ldots: f_{n}\right]$, where all components are homogeneous polynomials of the same degree and with no common zeroes except the origin.

Under the assumptions of Theorem 4.1. we will call the degree of the polynomials $f_{0}, \ldots, f_{n}$ the degree of the holomorphic map $f$.

The goal in this section is to prove the following theorem.

Theorem 4.2. Let $f: \mathbb{C} P(n) \rightarrow \mathbb{C} P(n)$ be holomorphic of degree $d \geq 2$.

(a) $f$ has infinitely many periodic orbits.

(b) There exists $M>0$ such that $p \in \operatorname{Per}(f)$ for all $p>M$ prime.

(c) If $f$ is transversal, then $\operatorname{Per}(f)=\mathbb{N}$.

We remark that part (a) was shown for $d=2$ in [10]. The proof is done from a complex analytic point of view.

We will prove Theorem 4.2 by computing the Lefschetz numbers and the periodic Lefschetz numbers of $f$.

Let $f: \mathbb{C} P(n) \rightarrow \mathbb{C} P(n)$ be a holomorphic map. In order to compute the Lefschetz numbers of $f$ we need to find its homology class. The following are well known facts from topology.

Proposition 4.3. The homology groups of $\mathbb{C} P(n)$ with rational coefficients are

$$
H_{i}(\mathbb{C} P(n), \mathbb{Q})= \begin{cases}\mathbb{Q} & \text { for } i=0,2,4, \ldots, 2 n, \\ 0 & \text { otherwise }\end{cases}
$$

Moreover, any continuous mapping $f$ of $\mathbb{C} P(n)$ induces homomorphisms on the homology groups

$$
f_{* 2 k}=\left(a^{k}\right)
$$

for $k=0,1, \ldots, n$ and for some $a \in \mathbb{Z}$ that depends on $f$.

For a proof see for example [20].

We now need to learn how the number $a$ depends on $f$. This follows from the fact that the last homology group of a compact manifold can be computed from the topological degree of the function. We state this result in a more general form below (see for example [13]). As usual, $\operatorname{Int}(A)$ denotes the interior of the set $A$.

Proposition 4.4. Let $f: M \rightarrow M$ be a $\mathcal{C}^{1}$ map and $M$ a compact, connected and oriented differentiable manifold of dimension $n$.

(a) If $q \in \operatorname{Int}(M)$ is a regular value of $f$, then $f^{-1}(q)$ is a finite number of points $p_{1}, \cdots, p_{k}$. 
(b) The group $H_{n}(M, \mathbb{Q})$ has one generator, and hence $f_{* n}$ is multiplication by an integer $D$, called the topological degree of $f$.

(c) Under the assumptions of the above statements, let $D_{i}=1$ (respectively -1 ) if $f$ preserves (respectively reverses) orientation at $p_{i}$. Then $D=\sum_{i=1}^{k} D_{i}$, and it is independent of the regular point $q$ chosen.

We apply this proposition to show

Proposition 4.5. Let $f: \mathbb{C} P(n) \rightarrow \mathbb{C} P(n)$ be a holomorphic map and let $d$ be its degree. Then, the homomorphisms induced by $f$ on the homology groups are

$$
f_{* 2 k}=\left(d^{k}\right) \text { for } k=0,1, \ldots, n .
$$

Hence,

$$
L(f)=\sum_{i=1}^{2 n}(-1)^{i} \operatorname{trace}\left(f_{* i}\right)=1+d+d^{2}+\cdots+d^{n} .
$$

Proof. Since we have seen that holomorphic maps always preserve orientation, the topological degree of $f$ is the number of preimages of a regular point. By Bézout's Theorem (see for example [10]), the number of preimages of a regular point is the product of the degrees of the polynomial coordinates, that is, $d^{n}$. Hence

$$
f_{* 2 n}=\left(d^{n}\right) .
$$

From Proposition 4.3 the result follows.

Proof of Theorem 4.2. Since the nonzero homology groups of $\mathbb{C} P(n)$ are one-dimensional, by using Proposition 4.5, we have that for any $m>0$

$$
L\left(f^{m}\right)=1+d^{m}+d^{2 m}+\ldots+d^{n m} .
$$

Since $d \geq 2$, the sequence $\left\{L\left(f^{m}\right)\right\}_{m \in \mathbb{N}}$ is unbounded, and hence (a) follows from Corollary 2.3.

Moreover, it is clear that

$$
d^{n m}<L\left(f^{m}\right)<d^{n m+1},
$$

and we can apply Proposition 3.5 with $k=n$ to conclude that $l\left(f^{m}\right) \neq 0$ for all $m \geq 3$. Also,

$$
l(f)=L(f)=1+d+\ldots+d^{n}
$$

and

$$
\begin{aligned}
l\left(f^{2}\right) & =L\left(f^{2}\right)-L(f) \\
& =1+d^{2}+d^{4}+\ldots+d^{2 n}-\left(1+d+\ldots+d^{n}\right) \\
& >P_{2 n}(d),
\end{aligned}
$$

where $P_{2 n}(d)$ is as in Lemma 3.6 and hence greater than 0 for $d \geq 2$. One concludes then that $l\left(f^{m}\right)>0$ for all $m \in \mathbb{N}$. Statements (b) and (c) follow then from 3.1 
4.2. Holomorphic maps on some Hopf surfaces. A compact complex surface $X$ is called a Hopf surface if its universal covering is biholomorphically equivalent to $\mathbb{C}^{2} \backslash\{0\}$ and its fundamental group equals $\mathbb{Z}$.

The group of covering transformations of a Hopf surface is generated by a contraction

$$
M: \mathbb{C}^{2} \rightarrow \mathbb{C}^{2}, \quad M(0)=0,
$$

which, after a change of coordinates, can always be written as

$$
M(z, w)=\left(\alpha z+\lambda w^{p}, \beta w\right)
$$

where $p \in \mathbb{N} \backslash\{0\}$, and $\alpha, \beta, \lambda \in \mathbb{C}$ are subject to

$$
0<|\alpha| \leq|\beta|<1 \text { and }\left(\alpha-\beta^{p}\right) \lambda=0 .
$$

The Hopf surface $X$ defined by a particular $M(z, w)$ is the space $\mathbb{C}^{2} \backslash\{0\}$ after identifying points that belong to the same orbit under $M$. Holomorphic maps from $X$ to $X$ are holomorphic maps $f: \mathbb{C}^{2} \backslash\{0\} \rightarrow \mathbb{C}^{2} \backslash\{0\}$ that send orbits of $M$ to orbits of $M$; that is,

$$
f \circ M=M^{d} \circ f, \quad \text { for a unique } d \in \mathbb{N} .
$$

There is a classification of Hopf surfaces that depends on the values of $\alpha, \beta, \lambda$ and $p$ (see for example [21]). In this subsection we deal with Hopf surfaces that are generated by a contraction of the type

$$
M(z, w)=\left(\begin{array}{cc}
\alpha & 1 \\
0 & \alpha
\end{array}\right)\left(\begin{array}{c}
z \\
w
\end{array}\right),
$$

where $0<|\alpha|<1$. From now on and unless otherwise specified, when we refer to a Hopf surface we mean a surface of this type.

Proposition 4.6. Let $X$ be a Hopf surface and $f: X \rightarrow X$ a holomorphic map. Let $d$ be the positive integer for which $f \circ M=M^{d} \circ f$. Then,

$$
f(z, w)=\left(a w^{d}+d b z w^{d-1}, b w^{d}\right),
$$

for some $a \in \mathbb{C}$ and $b \in \mathbb{C} \backslash\{0\}$.

The proof is very technical, and it is postponed to the appendix.

In an analogous way to the case of $\mathbb{C} P(n)$, in this section we prove the following theorem.

Theorem 4.7. Let $X$ be a Hopf surface and $f: X \rightarrow X$ a holomorphic map as in the statement of Proposition 4.6 , with $d \geq 2$. Then the following statements are satisfied.

(a) $f$ has infinitely many periodic orbits.

(b) There exists $M>0$ such that $p \in \operatorname{Per}(f)$ for all $p>M$ prime.

(c) If $f$ is transversal, then $\operatorname{Per}(f)=\mathbb{N}$.

As in the application to $\mathbb{C} P(n)$, we will show that the periodic Lefschetz numbers are all different from 0 .

Since every Hopf surface $X$ is homeomorphic to $S^{1} \times S^{3}$, we have

$$
H_{i}(X, \mathbb{Q})= \begin{cases}\mathbb{Q} & \text { if } i=0,1,3,4 \\ 0 & \text { otherwise }\end{cases}
$$


To compute $L\left(f^{m}\right)$ we need then to compute the homology class of $f$, that is, $f_{* 0}$, $f_{* 1}, f_{* 3}$ and $f_{* 4}$. Since $X$ is connected, we already know that $f_{* 0}=\mathrm{Id}$, hence $\operatorname{trace}\left(f_{* 0}\right)=1$. To compute the last one, we will use Proposition 4.4 .

Proposition 4.8. The topological degree of a holomorphic map as in Proposition 4.6 is $D=d^{2}$. Hence

$$
\operatorname{trace}\left(f_{* 4}\right)=d^{2}
$$

Proof. Recall that the topological degree of a holomorphic map $f$ is the number of preimages of any regular point, since $f$ preserves orientation at all points. In this case, for $\left(z_{0}, w_{0}\right)$ generic, the system

$$
\begin{aligned}
a w^{d}+d b z w^{d-1} & =z_{0}, \\
b w^{d} & =w_{0},
\end{aligned}
$$

has $d$ distinct solutions in $\mathbb{C}^{2}$. When we consider the map $f$ on $X$ we must do this $d$ times, i.e. we must look for points $(z, w)$ such that

$$
f(z, w)=M^{i}\left(z_{0}, w_{0}\right), \quad 0 \leq i<d .
$$

Those will be all distinct and there cannot be any others. Hence, by Proposition 4.4 we obtain $D=d^{2}$, and $f_{* 4}$ is multiplication by $d^{2}$.

To determine the remaining necessary traces for the computation of $L\left(f^{m}\right)$ is not at all trivial. However, one can use the Lefschetz numbers of $f$ and $f^{2}$ to compute them. First, we give an auxiliary lemma.

Lemma 4.9. A map $f$ as in Proposition 4.6 has $(d-1)^{2}$ fixed points and $\left(d^{2}-1\right)^{2}$ fixed points of $f^{2}$.

Proof. A point $(z, w)$ satisfying $f(z, w)=M^{n}(z, w)$ for some $n \geq 0$ is a fixed point of $f$. Those are the solutions of the following system:

$$
\begin{aligned}
a w^{d}+b d z w^{d-1} & =\alpha^{n} z+n \alpha^{n-1} w, \\
b w^{d} & =\alpha^{n} w .
\end{aligned}
$$

It is easy to check that there are $d-1$ solutions for each $n$.

Note that if $f(z, w)=M^{n}(z, w)$, then, for all $k \in \mathbb{N}$,

$$
f\left(M^{k}(z, w)\right)=M^{k d}(f(z, w))=M^{k d+n}(z, w)=M^{n+k(d-1)}\left(M^{k}(z, w)\right),
$$

where we have used the relation $f \circ M=M^{d} \circ f$. Hence, if $(z, w)$ is a solution of $f(z, w)=M^{n}(z, w)$, then its orbit by $M$ contains solutions of $f(z, w)=$ $M^{n+k(d-1)}(z, w)$ for all $k$ and not of any others. So the fixed points of $f$ are the solutions of

$$
f(z, w)=M^{n+i}(z, w), \quad 0 \leq i \leq d-2 .
$$

Since there are $d-1$ of these equations and we have seen that each has $d-1$ solutions, we obtain that $f$ has $(d-1)^{2}$ fixed points.

For the periodic points, observe that $f^{2}(z)$ is also a holomorphic map of $X$ where $d$ is replaced by $d^{2}$. The same argument as above gives the existence of $\left(d^{2}-1\right)^{2}$ fixed points.

Proposition 4.10. Let $X$ and $f$ be as in Theorem 4.7. Then

$$
\operatorname{trace}\left(f_{* 1}\right)=\operatorname{trace}\left(f_{* 3}\right)=d \text {. }
$$


Proof. Let $A=\operatorname{trace}\left(f_{* 1}\right)$ and $B=\operatorname{trace}\left(f_{* 3}\right)$. Then, by definition of the Lefschetz numbers,

$$
\begin{aligned}
1-A-B+d^{2} & =L(f), \\
1-A^{2}-B^{2}+d^{4} & =L\left(f^{2}\right) .
\end{aligned}
$$

Since $f$ is holomorphic and transversal, $L(f)$ (respectively $L\left(f^{2}\right)$ ) is the number of fixed points of $f$ (respectively of $f^{2}$ ). Since we have an expression for $f$, we can compute those.

Therefore, system (4.1) becomes

$$
\begin{aligned}
1-A-B+d^{2} & =(d-1)^{2}, \\
1-A^{2}-B^{2}+d^{4} & =\left(d^{2}-1\right)^{2},
\end{aligned}
$$

and its solution is $A=B=d$. This concludes the proof of the proposition.

Proof of Theorem 4.7. We now have an explicit expression for the Lefschetz numbers:

$$
L\left(f^{m}\right)=d^{2 m}-2 d^{m}+1 .
$$

Since $d \geq 2$, the sequence $\left\{L\left(f^{m}\right)\right\}_{m \in \mathbb{N}}$ is unbounded. Hence, statement (a) follows from Corollary 2.3.

We observe that

$$
d^{2 m-1}<L\left(f^{m}\right)<d^{2 m} .
$$

Hence, by Proposition 3.5 (see Remark 3.7), $l\left(f^{m}\right)>0$ for all $m \in \mathbb{N}$. Statements (b) and (c) now follow from Theorem 3.1 .

\section{Another proof of BaKer's Theorem}

In 1964, Baker completely described the periodic set of any holomorphic map of the Riemann sphere, i.e., a rational map, and also in the special case of a polynomial.

Theorem 5.1 ([3]). (a) Let $P$ be a polynomial of degree at least two and suppose that $P$ has no periodic points of period $n$. Then $n=2$, and $P$ is conjugate to $z \mapsto z^{2}-z$.

(b) Let $R$ be a rational function of degree $d \geq 2$, and suppose that $R$ has no periodic points of period $n$. Then $(d, n)$ is one of the pairs $(2,2),(2,3),(3,2),(4,2)$.

The proof of this theorem can also be found in [4]. In this subsection we will give an alternative proof which uses the Lefschetz numbers. The main tool is the following theorem which is the analogue to Theorems 2.4 and 3.1 for general holomorphic maps of the Riemann sphere.

Theorem 5.2. (a) Let $P: \mathbb{C} \rightarrow \mathbb{C}$ be a polynomial of degree $d \geq 2$. If $l\left(P^{m}\right) / m>$ $d-1$, then $m \in \operatorname{Per}(P)$.

(b) Let $R: \mathbb{C} \rightarrow \mathbb{C}$ be a rational map of degree $d \geq 2$. If $l\left(R^{m}\right) / m>2(d-1)$, then $m \in \operatorname{Per}(R)$.

The proof follows the idea of the proof of Theorem 2.4. Thus, by using the two definitions of the Lefschetz numbers we will find two expressions for the dynamical zeta function. From their comparison the result will follow. 
Let $R$ be a rational function of degree $d$. Let PO be the set of periodic orbits of $R$. Given a periodic orbit $\gamma \in \mathrm{PO}$, let $p(\gamma)$ be its period. If $\gamma=\left\{z_{p(\gamma)}=\right.$ $\left.z_{0}, z_{1}, \ldots, z_{p(\gamma)-1}\right\}$ with $z_{i}=R^{i}\left(z_{0}\right)$, we define the multiplier of $\gamma$ as

$$
\rho=\rho(\gamma)=\left(R^{p(\gamma)}\right)^{\prime}\left(z_{0}\right)=\prod_{i=0}^{p(\gamma)-1} R^{\prime}\left(z_{i}\right)
$$

With this notation, $R$ is transversal if and only if there is no periodic orbit whose multiplier is a root of unity. Since we allow $R$ not to be transversal, we must account for this kind of periodic orbit.

The first step is to find an expression for the Poincaré index of $R^{m}$ at any periodic point. Let $\mathrm{PO}^{N T} \subset \mathrm{PO}$ be the set of periodic orbits whose multiplier is a root of unity. Then, if $\gamma \in \mathrm{PO} \backslash \mathrm{PO}^{N T}$,

$$
\operatorname{ind}\left(R^{m}, z\right)=1 \quad \text { for all } z \in \gamma \text { and for all } m \in \mathbb{N} \text {. }
$$

Now take $\gamma \in \mathrm{PO}^{N T}$ with multiplier $\rho(\gamma)=\exp (2 \pi i r / s)$ with $r$ and $s$ coprime, and assume for the moment that $\gamma$ is a fixed point $z_{0}$. The index of $R^{m}$ at $z_{0}$ will be different from 1 only for those $m$ for which $\left(R^{m}\right)^{\prime}\left(z_{0}\right)=1$. That is,

$$
\operatorname{ind}\left(R^{m}, z_{0}\right)=1 \text { for all } m \text { such that } s \not X m \text {. }
$$

The dynamics around a nontransversal fixed point are described by the Leau-Fatou Flower Theorem. We state this theorem below, together with a well known fact of complex dynamics (see for example [4]).

Theorem 5.3. Let $R$ be a rational map and $z_{0}$ a fixed point of $R$ with multiplier $\exp (2 \pi i r / s)$, where $r$ and $s$ are coprime. Then, there exists $k \in \mathbb{N}$ such that for all $l \in \mathbb{N}$

$$
R^{l s}(z)=\left(z-z_{0}\right)+\left(z-z_{0}\right)^{k s+1}+\text { h.o.t. }
$$

Moreover,

(a) there are exactly $2 k s$ elliptic sectors around $z_{0}$, and

(b) there are $k$ critical points (points of derivative equal to 0) attracted to $z_{0}$.

By the Bendixson Index Formula (see 14]), we have

$$
\operatorname{ind}\left(R^{l s}, z_{0}\right)=k s+1 \text { for some } k \in \mathbb{N} \text { and for all } l \in \mathbb{N} \text {. }
$$

Returning to periodic orbits, let $\gamma=\left\{z_{0}, \ldots, z_{p(\gamma)-1}\right\}$ be a periodic orbit of period $p(\gamma)$ and multiplier $\rho(\gamma)=\exp (2 \pi i r / s(\gamma))$. Applying Theorem 5.3 to $R^{p(\gamma)}$, we obtain a natural number $k=k(\gamma) \in \mathbb{N}$ such that

$R^{l p(\gamma) s(\gamma)}\left(z_{i}\right)=\left(z-z_{i}\right)+\left(z-z_{i}\right)^{k(\gamma) s(\gamma)+1}+h . o . t$. for all $l \in \mathbb{N}$ and $0 \leq i<p(\gamma)$.

Then

$$
\operatorname{ind}\left(R^{m}, z_{i}\right)= \begin{cases}k(\gamma) s(\gamma)+1 & \text { if } m=l p(\gamma) s(\gamma) \text { for some } l \in \mathbb{N} \\ 1 & \text { otherwise. }\end{cases}
$$

Proposition 5.4. Let $R$ be a rational map of degree $d \geq 2$. Then the dynamical zeta function of $R$ can be written as

$$
Z_{R}(t)=\prod_{\gamma \in \mathrm{PO}}\left(1-t^{p(\gamma)}\right)^{-1} \prod_{\gamma \in \mathrm{PO}^{N T}}\left(1-t^{p(\gamma) s(\gamma)}\right)^{-k(\gamma)}
$$


Proof. This proof uses ideas of [8]. For $\gamma \in$ PO let $L_{m}(R, \gamma)$ be the contribution of $\gamma$ to $L\left(R^{m}\right)$, i.e.

$$
L_{m}(R, \gamma)= \begin{cases}0 & \text { if } p(\gamma) \chi m \\ \sum_{z \in \gamma} \operatorname{ind}\left(R^{m}, z\right) & \text { if } p(\gamma) \mid m\end{cases}
$$

Then

$$
L\left(R^{m}\right)=\sum_{z \in \operatorname{Fix}\left(R^{m}\right)} \operatorname{ind}\left(R^{m}, z\right)=\sum_{\gamma \in \mathrm{PO}} L_{m}(R, \gamma) .
$$

For $\gamma \in \mathrm{PO} \backslash \mathrm{PO}^{N T}$ we have

$$
L_{m}(R, \gamma)= \begin{cases}0 & \text { if } p(\gamma) \backslash m \\ p(\gamma) & \text { otherwise }\end{cases}
$$

and, if $\gamma \in \mathrm{PO}^{N T}$, then

$$
L_{m}(R, \gamma)= \begin{cases}0 & \text { if } p(\gamma) \chi m, \\ p(\gamma) & \text { if } p(\gamma) \mid m \text { but } s(\gamma) \chi m, \\ p(\gamma)(k(\gamma) s(\gamma)+1) & \text { if } p(\gamma) \mid m \text { and } s(\gamma) \mid m .\end{cases}
$$

Therefore,

$$
\begin{aligned}
Z_{R}(t) & =\exp \left(\sum_{m=1}^{\infty} \frac{L\left(R^{m}\right)}{m} t^{m}\right) \\
& =\exp \left(\sum_{m=1}^{\infty} \frac{\sum_{\gamma \in \mathrm{PO}} L_{m}(R, \gamma)}{m} t^{m}\right) \\
& =\exp \left(\sum_{\gamma \in \mathrm{PO} m=1}^{\infty} \frac{L_{m}(R, \gamma)}{m} t^{m}\right) \\
& =\exp \left(\sum_{\gamma \in \mathrm{PO} l=1}^{\infty} \frac{L_{l p(\gamma)}(R, \gamma)}{l p(\gamma)} t^{l p(\gamma)}\right) \\
& =\exp \left(\sum_{\gamma \in \mathrm{PO} l=1}^{\infty} \sum_{l p}^{\infty} \frac{p(\gamma)}{l p(\gamma)} t^{l p(\gamma)}+\sum_{\gamma \in \mathrm{PO}^{N T}} \sum_{l=1}^{\infty} \frac{p(\gamma) k(\gamma) s(\gamma)}{l p(\gamma) s(\gamma)} t^{l p(\gamma) s(\gamma)}\right) \\
& =\prod_{\gamma \in \mathrm{PO}} \exp \left(-\log \left(1-t^{p(\gamma)}\right)\right) \prod_{\gamma \in \mathrm{PO}^{N T}} \exp \left(-k(\gamma) \log \left(1-t^{p(\gamma) s(\gamma)}\right)\right) \\
& \prod_{\gamma \in \mathrm{PO}}\left(1-t^{p(\gamma)}\right)^{-1} \prod_{\gamma \in \mathrm{PO}^{N T}}\left(1-t^{p(\gamma) s(\gamma)}\right)^{-k(\gamma)} .
\end{aligned}
$$

The second expression for the dynamical zeta function of $R$ uses the definitions of the Lefschetz numbers in terms of homology. With some computations, one can show (see for instance [2]) that

$$
Z_{R}(t)=\prod_{m=1}^{\infty}\left(1-t^{m}\right)^{-\frac{l\left(f^{m}\right)}{m}} .
$$

Finally, the last ingredient in the proof of Theorem [5.2] is the second part of Theorem [5.3. By the Riemann-Hurwitz formula (see for instance [18]), a rational map can have at most $2(d-1)$ critical points. Clearly, a polynomial can have at 
most $d-1$ critical points. By Theorem [5.3, this translates into the following upper bounds:

$$
\sum_{\gamma \in \mathrm{PO}^{N T}} k(\gamma) \leq \begin{cases}d-1 & \text { if } R \text { is a polynomial, } \\ 2(d-1) & \text { otherwise. }\end{cases}
$$

Proof of Theorem 5.2. Assume $P$ is a polynomial and $l\left(P^{m}\right) / m>d-1$. Then, by (5.1), the zeta function has more than $d-1$ factors of the form $1-t^{m}$. On the other hand, by Proposition 5.4

$$
Z_{R}(t)=\prod_{\gamma \in \mathrm{PO}}\left(1-t^{p(\gamma)}\right)^{-1} \prod_{\gamma \in \mathrm{PO}^{N T}}\left(1-t^{p(\gamma) s(\gamma)}\right)^{-k(\gamma)} .
$$

Since $\sum_{\gamma \in \mathrm{PO}^{N T}} k(\gamma) \leq d-1$, it follows that there can be at most $d-1$ factors of the form $1-t^{m}$ coming from the second product, for values of $p$ and $s$ such that $p s=m$. This implies that at least one of them must come from the first product. That is, there is at least one orbit $\gamma$ such that $p(\gamma)=m$.

For a general rational function, apply the same argument, replacing $d-1$ by $2(d-1)$. The second part of the theorem follows.

We now apply Theorem 5.2 to prove Baker's Theorem.

Proof of Theorem 5.1. Recall that for a rational map $R, L\left(R^{m}\right)=1+d^{m}$. Since $\sum_{r \mid m} \mu(r)=0$, we have

$$
l\left(R^{m}\right)=d^{m}-\widetilde{P}_{k}(d),
$$

where $\widetilde{P}_{k}(d)$ is a polynomial with coefficients equal to 1 or -1 and no constant term. Let

$$
P_{k}(d)=d^{k}+d^{k-1}+\ldots+d .
$$

Then $l\left(R^{m}\right) \geq d^{m}-P_{k}(d)$, where $k \leq m / 2$. By induction it is easy to see that

$$
d^{m}-P_{k}(d)>2 m(d-1), \text { for all } m \geq 4 \text { and } d \geq 2 .
$$

Hence rational functions, and, in particular, polynomials, have periodic orbits of all periods greater than or equal to 4 .

We now compute

$$
\begin{array}{lll}
l\left(R^{2}\right)=d^{2}-d & >2(d-1) & \text { if } d>2, \\
l\left(R^{2}\right)=d^{2}-d & >4(d-1) & \text { if } d>4, \\
l\left(R^{3}\right)=d^{3}-d & >6(d-1) & \text { if } d>2,
\end{array}
$$

and thus, from Theorem 5.2 , we obtain the remaining cases.

\section{Appendix: Proof of Proposition 4.6}

We restate the proposition for convenience.

Proposition 4.6. Let $X$ be a Hopf surface and $f: X \rightarrow X$ a holomorphic map. Let $d$ be the positive integer for which $f \circ M=M^{d} \circ f$. Then,

$$
f(z, w)=\left(a w^{d}+d b z w^{d-1}, b w^{d}\right),
$$

for some $a \in \mathbb{C}$ and $b \in \mathbb{C} \backslash\{0\}$. 
Proof. Since $M(z, w)=(\alpha z+w, \alpha w)$, if $f(z, w)=\left(f_{1}(z, w), f_{2}(z, w)\right)$, then

$$
\begin{aligned}
& f_{1}(\alpha z+w, \alpha w)=\alpha^{d} f_{1}(z, w)+d \alpha^{d-1} f_{2}(z, w), \\
& f_{2}(\alpha z+w, \alpha w)=\alpha^{d} f_{2}(z, w) .
\end{aligned}
$$

By Hartogs' Theorem (see for example [22]), $f$ extends to a holomorphic mapping of $\mathbb{C}^{2}$ with $f(0)=0$, and hence it can be expanded into its Taylor series around 0 . We will expand the functions on both sides and compare the coefficients of the same degree to get, by induction, the conditions on $f$. We start with $f_{2}$.

Suppose $f_{2}(z, w)=\sum_{k, j \in \mathbb{N}} b_{k, j} z^{k} w^{j}$. Then, equation (A.3) becomes

$$
\sum_{k, j \in \mathbb{N}} b_{k, j}(\alpha z+w)^{k} \alpha^{j} w^{j}=\alpha^{d} \sum_{k, j \in \mathbb{N}} b_{k, j} z^{k} w^{j}
$$

Since

$$
(\alpha z+w)^{k}=\sum_{i=0}^{k}\left(\begin{array}{l}
k \\
i
\end{array}\right) \alpha^{i} z^{i} w^{k-i},
$$

the left hand side of (A.4) becomes

$$
\begin{aligned}
\sum_{k, j \in \mathbb{N}} b_{k, j}(\alpha z+w)^{k} \alpha^{j} w^{j} & =\sum_{p \in \mathbb{N}} z^{p} \sum_{j \in \mathbb{N}} \sum_{k \in \mathbb{N}} b_{k, j}\left(\begin{array}{c}
k \\
p \geq p
\end{array}\right) \alpha^{p+j} w^{k+j-p} \\
& =\sum_{p \in \mathbb{N}} z^{p} \sum_{i, j \in \mathbb{N}} b_{i+p, j}\left(\begin{array}{c}
p+i \\
p
\end{array}\right) \alpha^{p+j} w^{i+j} \\
& =\sum_{p, q \in \mathbb{N}} z^{p} w^{q}\left(\sum_{i+j=q} b_{i+p, j}\left(\begin{array}{c}
p+i \\
p
\end{array}\right) \alpha^{p+j}\right) \\
& =\sum_{p, q \in \mathbb{N}} z^{p} w^{q}\left(\sum_{i=0}^{q} b_{p+i, q-i}\left(\begin{array}{c}
p+i \\
p
\end{array}\right) \alpha^{p+q-i}\right) .
\end{aligned}
$$

Comparing the coefficients of the same degree in (A.4), we get the equations

$$
\sum_{i=0}^{q} b_{p+i, q-i}\left(\begin{array}{c}
p+i \\
p
\end{array}\right) \alpha^{p+q-i}=\alpha^{d} b_{p, q}
$$

or, equivalently,

$$
b_{p, q}\left(\alpha^{p+q}-\alpha^{d}\right)+b_{p+1, q-1}(p+1) \alpha^{p+q-1}+\ldots+b_{p+q, 0}\left(\begin{array}{c}
p+q \\
p
\end{array}\right) \alpha^{p}=0 .
$$

We claim that $b_{p, q}=0$ for all $(p, q) \neq(0, d)$. We show this claim by induction on $q$. For each $d \geq 1$, we treat the cases $q \leq d$ and $q>d$ separately.

Case $q \leq d$. For $q=0$, equation (A.5) reads $b_{p, 0}\left(\alpha^{p}-\alpha^{d}\right)=0$.

Hence $b_{p, 0}=0$ for all $p \neq d$. But for $q=1$ and $p=d-1$ we have

$$
b_{d-1,1}\left(\alpha^{d}-\alpha^{d}\right)+b_{d, 0} d \alpha^{d-1}=0 .
$$

Hence $b_{d, 0}=0$.

We now assume $b_{p, q-1}=b_{p, q-2}=\cdots=b_{p, 0}=0$ for all $p$. Then, equation (A.5) becomes $b_{p, q}\left(\alpha^{p+q}-\alpha^{d}\right)=0$. Hence, $b_{p, q}=0$ unless $p+q=d$. In particular, $b_{0, q}=0$ unless $q=d$. For $p+q=d$ and $p \geq 1$ we have $b_{p-1, q+1}\left(\alpha^{d}-\alpha^{d}\right)+b_{p, q} p \alpha^{d-1}=0$, and therefore $b_{p, q}=0$. 
Case $q=d+i$ with $i>0$. If $i=1$ eq. (A.5 says $b_{p, d+1}\left(\alpha^{p+d+1}-\alpha^{d}\right)$ $+b_{p+1, d}(p+1) \alpha^{p+d}=0$. Since $p+1>0$, this implies that $b_{p+1, d}=0$, and consequently $b_{p, d+1}=0$ unless $p+d+1=d$, which is impossible.

Suppose $b_{p, q}=0$ for all $p \geq 0$ and $d+1 \leq q<d+i$. Then, again from (A.5) we obtain that $b_{p, d+i}\left(\alpha^{p+d+i}-\alpha^{d}\right)=0$. Hence $b_{p, d+i}=0$ unless $p+d+i=d$, which is impossible.

This concludes the proof of the claim, and we have shown that

$$
f_{2}(z, w)=b w^{d} \quad \text { for some } b \in \mathbb{C} .
$$

We note that $b$ must be different from zero. Indeed, if that were not the case, the same calculations applied to $f_{1}(z, w)$ would lead to $f_{1}(z, w)=a w^{d}$ for some $a \in \mathbb{C}$, and consequently $f^{2}(z, w) \equiv(0,0) \notin X$.

To find the expression for $f_{1}(z, w)$, assume that $f_{1}(z, w)=\sum_{i, j \in \mathbb{N}} a_{i, j} z^{i} w^{j}$. Then, repeating the above arguments, we see that equation (A.2) becomes

$$
\sum_{p, q \in \mathbb{N}} z^{p} w^{q}\left(\sum_{i=0}^{q} a_{p+i, q-i}\left(\begin{array}{c}
p+i \\
p
\end{array}\right) \alpha^{p+q-i}\right)=\alpha^{d} \sum_{p, q \in \mathbb{N}} a_{p, q} z^{p} w^{q}+d \alpha^{d-1} b w^{d} .
$$

For $p=0$ and $q=d$ the terms with $a_{0, d}$ cancel out, and we have

$$
a_{1, d-1} \alpha^{d-1}+a_{2, d-2} \alpha^{d-2}+\ldots+a_{d, 0}=d \alpha^{d-1} b .
$$

Otherwise, as before, we get the equation equivalent to (A.5), i.e.

$$
a_{p, q}\left(\alpha^{p+q}-\alpha^{d}\right)+a_{p+1, q-1}(p+1) \alpha^{p+q-1}+\ldots+a_{p+q, 0}\left(\begin{array}{c}
p+q \\
p
\end{array}\right) \alpha^{p}=0 .
$$

We claim that $a_{p, q}=0$ for all $(p, q) \notin\{(0, d),(1, d-1)\}$. We will show this claim in four steps.

Case $q<d-1$ with $d \geq 2$. Then $a_{p, q}=0$ for all $p \geq 0$. If $q=0$, by equation (A.7) we get $a_{p, 0}\left(\alpha^{p}-\alpha^{d}\right)=0$. Hence $a_{p, 0}=0$ unless $p=d$. But we have $a_{d-1,1}\left(\alpha^{d}-\alpha^{d}\right)+a_{d, 0} d \alpha^{d-1}=0$, and therefore $a_{d, 0}=0$.

Suppose it is true until $q-1$. Then, from (A.7) we have that $a_{p, q}\left(\alpha^{p+q}-\alpha^{d}\right)=0$. So $a_{p, q}=0$ unless $p=d-q$. But $a_{d-q-1, q+1}\left(\alpha^{d}-\alpha^{d}\right)+a_{d-q, q} \alpha^{d-1}=0$, and therefore $a_{d-q, q}=0$.

Case $q=d-1$. If $p \neq 1$, then $a_{p, d-1}=0$ and $a_{1, d-1}=b d$. From equation A.6 we have $a_{p, d-1}\left(\alpha^{p+d-1}-\alpha^{d}\right)=0$. Hence $a_{p, d-1}=0$ unless $p=1$. On the other hand, from equation (A.7) we obtain $a_{0, d}\left(\alpha^{d}-\alpha^{d}\right)+a_{1, d-1} \alpha^{d-1}=d \alpha^{d-1} b$. Therefore $a_{1, d-1}=d b$.

Case $q=d$. If $p \neq 0$, then $a_{p, d}=0$. This comes from $a_{p, d}\left(\alpha^{p+d}-\alpha^{d}\right)+a_{p+1, d-1}$ $=0$ and the case $q=d-1$.

Case $q>d$. Then $a_{p, q}=0$ for all $p>0$. If $q=d+1$, we have

$$
a_{p, d+1}\left(\alpha^{p+d+1}-\alpha^{d}\right)+a_{p+1, d}=0 .
$$

From the case $q=d$ and the fact that $p+d+1 \neq d$ we obtain $a_{p, d+1}=0$ for all $p>0$.

Suppose it is true for $q=d+i-1$. For $q=d+i$ we have $a_{p, d+i}\left(\alpha^{p+d+i}-\alpha^{d}\right)=0$, and therefore $a_{p, d+i}=0$ since $p+d+i \neq d$.

These four steps conclude the proof of the claim. We have shown that

$$
f_{1}(z, w)=a w^{d}+d b z w^{d-1}
$$

for $a, b \in \mathbb{C}, b \neq 0$. This concludes the proof of the proposition. 


\section{REFERENCES}

1. V. I. Arnold, Aspects des systèmes dynamiques, Preprint Ecole Polytechnique, Journées XUPS 1994. Also in Topological Methods in Nonlinear Analysis, Torum, 1994.

2. I. K. Babenko \& S. A. Bogatyi, The behavior of the index of periodic points under iterations of a mapping, Math. USSR Izvestiya 38 (1992), 1-26. MR 93a:58139

3. I. N. Baker, Fixpoints of polynomials and rational functions, J. London Math. Soc. 39 (1964), 615-622. MR 30:230

4. A. Beardon, Iteration of Rational Functions, Graduate Texts in Math., Springer-Verlag, 1991. MR 92j:30026

5. R. F. Brown, The Lefschetz fixed point theorem, Scott, Foresman and Company, Glenview, IL, 1971. MR 44:1023

6. J. Cronin, Differential Equations. Introduction and Qualitative Theorey, 2nd ed., Marcel Decker, Inc., 1994. MR 95h:34001

7. A. Dold, Fixed point indices of iterated maps, Invent. Math. 74 (1983), 419-435. MR 85c:54077

8. J. Franks, Homology and dynamical systems, CBMS Regional Conf. Ser. in Math., no 49, Amer. Math. Soc., Providence, R.I., 1982. MR 84f:58067

9. D. Fried, Periodic points of holomorphic maps, Topology 25 (1986), 429-441. MR 88:58168

10. J. E. Fornaess \& N. Sibony, Complex dynamics in higher dimension I, Astérisque 222 (1994), 201-231. MR 95i:32036

11. J. Guaschi \& J. Llibre, Periodic points of $\mathcal{C}^{1}$ maps and the asymptotic Lefschetz numbers, Int. J. of Bif. and Chaos Appl. Sci. Engrg. 5 (1995), 1369-1373. MR 96k:58178

12. A. Guillamon, X. Jarque, J. Llibre, J. Ortega and J. Torregrosa, Periods for transversal maps via Lefschetz numbers for periodic points, Trans. Amer. Math. Soc. 347 (1995), 4779-4806. MR 96c:58140

13. A. Kosinski, Differential Manifolds, Academic Press Inc., 1993. MR 95b:57001

14. S. Lefschetz, Differential Equations: Geometric Theory, Interscience, New York, 1963. MR 27:3864

15. J. Llibre, Lefschetz numbers for periodic points, Contemporary Math. 152 (1993), 215-227. MR 94g:55002

16. T. Matsuoka, The number of periodic points of smooth maps, Erg. Th. \& Dyn. Sys. 9 (1989), 153-163. MR 90g:58100

17. I. Niven \& H. S. Zuckerman, An introduction to the theory of numbers, fourth edition, John Wiley \& Sons, New York, 1980. MR 81g:10001

18. N. Steinmetz, Rational iteration: complex analytic dynamical systems, De Gruyter Studies in Mathematics, De Gruyter, 1993. MR 94h:30035

19. M. Shub and D. Sullivan, A remark on the Lefschetz fixed point formula for differentiable maps, Topology 13 (1974), 189-191. MR 50:3274

20. J. W. Vick, Homology Theory, 2nd ed., Springer-Verlag, 1994. MR 94i:55002

21. J. Wehler, Versal deformations of Hopf surfaces, J. fur die Reine und Ang. Math. (Journal de Crelle) 328 (1981), 22-32. MR 84h:32025

22. H. Whitney, Complex Analytic Varieties, Addison-Wesley Series in Mathematics, 1972. MR 52:8473

Departament de Matemàtiques, Universitat Autònoma de Barcelona, 08193 Bellaterra, Barcelona, Spain

Current address: Departament de Matemàtica Aplicada i Anàlisi, Gran Via 585, 08007 Barcelona, Spain

E-mail address: fagella@maia.ub.es

$U R L:$ www.maia.ub.es $/ \sim$ fagella

Departament de Matemàtiques, Universitat Autònoma de Barcelona, 08193 BellaTERRA, BARCELONA, SPAin

E-mail address: jllibre@mat.uab.es 